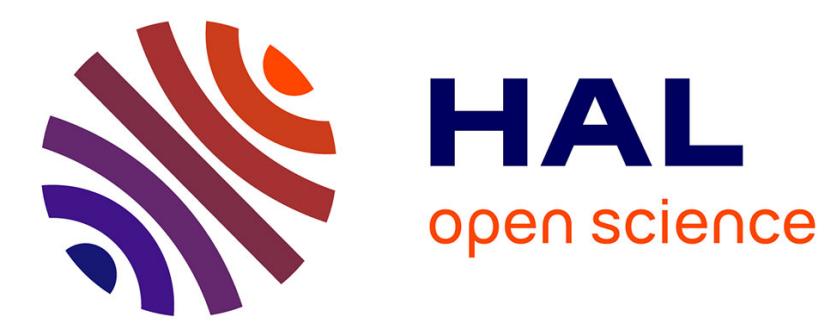

\title{
Extraordinary transmission beyond the cut-off through sub- $\lambda$ annular aperture arrays
}

\author{
F.I. Baida, Y. Poujet, J. Salvi, D. van Labeke, B. Guizal
}

\section{To cite this version:}

F.I. Baida, Y. Poujet, J. Salvi, D. van Labeke, B. Guizal. Extraordinary transmission beyond the cut-off through sub- $\lambda$ annular aperture arrays. Optics Communications, 2009, 282 (7), pp.1463-1466. 10.1016/j.optcom.2008.12.021 . hal-00361706

\section{HAL Id: hal-00361706 \\ https://hal.science/hal-00361706}

Submitted on 6 May 2021

HAL is a multi-disciplinary open access archive for the deposit and dissemination of scientific research documents, whether they are published or not. The documents may come from teaching and research institutions in France or abroad, or from public or private research centers.
L'archive ouverte pluridisciplinaire HAL, est destinée au dépôt et à la diffusion de documents scientifiques de niveau recherche, publiés ou non, émanant des établissements d'enseignement et de recherche français ou étrangers, des laboratoires publics ou privés. 


\title{
Extraordinary transmission beyond the cut-off through sub- $\lambda$ annular aperture arrays
}

\author{
Fadi I. Baida*, Yannick Poujet, Jérôme Salvi, Daniel Van Labeke, Brahim Guizal \\ Institut FEMTO-ST, Département d'Optique P.M. Duffieux,16 Route de Gray, 25030 Besançon, France
}

It was recently established that coaxial nano-aperture arrays perforated in optically thick silver films exhibit an extraordinary transmission in the visible range thanks to a guided mode. However this mode has a cut-off wavelength beyond which the transmission dramatically decreases. We propose here a cas-caded structure that is shown to act as a high quality spectral filter in the visible range (finesse up to 200). It is also found that the transmission is enhanced by a factor of 150 for specific wavelengths. This kind of cascaded nano-structured metallic layers offers many promising applications in nanophotonics such as the integration of functionalities for optical signal processing. It opens up the path for the conception of a new generation of integrated components based on metallo-dielectric structures that can be easily tai-lored as tunable devices.

\section{Introduction}

Extraordinary transmission through subwavelength apertures engraved in metallic films is a wide area of research. Transmission through annular apertures arrays (AAA) is a sub-domain of these researches and $90 \%$ transmission in the visible range can be achieved with sub- $\lambda$ coaxial apertures arranged into a square lattice $[1,2]$. This large transmission is due to one identified guided mode ( $\mathrm{TE}_{11}$-like mode) propagating inside each cavity [3]. The structure is a low-pass filter for wavelengths smaller than a cutoff $\left(\lambda_{c}^{\mathrm{TE}}{ }_{11}\right)$. The TEM mode has no cut-off but it can not be excited under normal incidence. A typical transmission spectrum is presented in Fig. 1. The guided mode, excited by the diffraction of the linearly polarized incident beam, can propagate inside the cavity with very weak losses in spite of the real nature of the metal used. A lot of studies are devoted to the fabrication and the characterization of AAA structures made in silver, gold or other metals [4-8].

What is important to note in Fig. 1 is that the position of the main transmission peak (located at the cut-off wavelength of the $\mathrm{TE}_{11}$-like mode of the infinite coaxial waveguide) depends both on the geometrical parameters of the annular aperture and on the nature of the metal used. It is almost independent of the metal thickness. Thus, if we want to increase the value of the cut-off, we should expand the outer radius (or simultaneously the inner one) of the aperture which is obviously limited by the distance between two apertures, i.e. by the period of the structure. In addition, this last value has to be chosen in order to cancel any surface plasmon

\footnotetext{
* Corresponding author.

E-mail address: fbaida@univ-fcomte.fr (F.I. Baida).
}

resonance near the cut-off [9] because the latter plays a negative role on the transmission obtained through guided modes [10]. As a consequence, the period must be smaller than the cut-off wavelength (for normal incidence).

To bypass this restraint, we propose to use a cascaded structure composed of two identical perforated metallic layers (cf. Fig. 2). Fabry-Pérot (FP) cavities using metallic meshes instead of the usual mirrors have been proposed in the late sixties in order to fabricate low-pass and band-pass filters in the far infrared (see review article [11]). This idea has also been recently applied in the infrared range for holes [12] or slits [13] arrays in metallic films. In our case dealing with the visible range, each layer consists of a square lattice of annular apertures of period $p=300 \mathrm{~nm}$, inner radius $R_{i}=$ $75 \mathrm{~nm}$ and outer radius $R_{o}=100 \mathrm{~nm}$ made in a $h=100 \mathrm{~nm}$ thick silver film.

\section{FDTD simulations}

In the following, the distance between the two layers is named $d$ and $\varepsilon$ is the dielectric constant of the intermediate medium.

To simplify the discussion, we consider a self-suspended structure where both the intermediate medium and the coaxial apertures are filled by air $(\varepsilon=1)$. Furthermore, we suppose that there is no lateral shift between the two structures, the apertures are then one in front of the other $\left(L_{x}=L_{y}=0\right.$ in Fig. 2). These two assumptions, that are not restrictive at all, are taken in order to have a simple explanation of the theoretical results.

The numerical study is done with a home-made finite difference time domain (FDTD) code. It includes a non uniform meshing in order to fulfill an accurate description of the fine details of the structure. The parameters of the calculations are given in the figure 


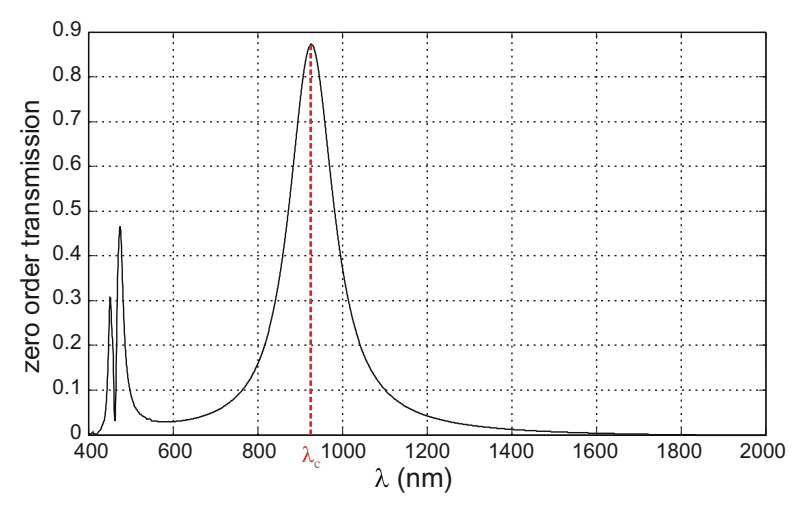

Fig. 1. Transmission spectrum through a single silver layer perforated with annular apertures arranged into a square array. The period is $p=350 \mathrm{~nm}$, the inner and the outer radii of the coaxial cavities are $R_{i}=75$ and $R_{o}=100 \mathrm{~nm}$, respectively. The metal thickness is set to $h=100 \mathrm{~nm}$ and the structure is illuminated by a linearly polarized plane wave at normal incidence. The spatial step of the FDTD algorithm was fixed to $5 \mathrm{~nm}$ for the metal structure and $25 \mathrm{~nm}$ elsewhere. The metal dispersion $\left(\varepsilon_{m}(\omega)\right)$ was also taken into account by a Drude's model as in Ref. [1]. Because of the spatial discretization, all geometrical parameters are defined with $5 \mathrm{~nm}$ uncertainty.

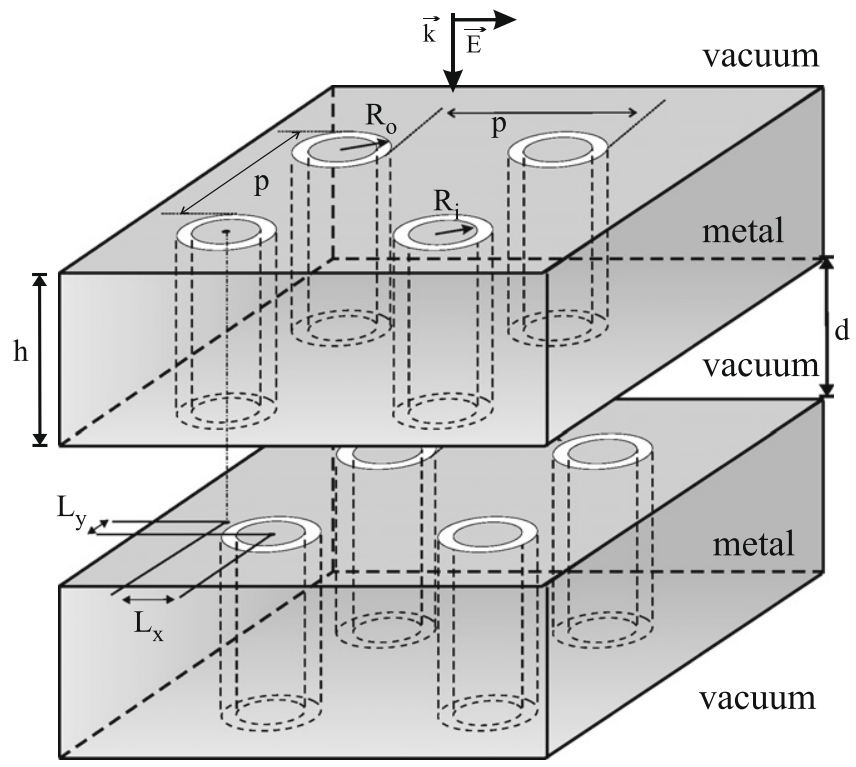

Fig. 2. Schematic view of the studied structure. As mentioned in Fig. 1, the period is noted $p$, the inner and the outer radius of the coaxial cavity are $R_{i}$ and $R_{o}$, respectively. The metal thickness is $h$, the distance between the two metallic layers is $d$ and the structure is illuminated by a linearly polarized plane wave at normal incidence. $L_{x}$ and $L_{y}$ are the lateral shifts along the $x$ and $y$ directions, respectively.

captions. Note that throughout this study, the illumination parameters are kept the same as in Fig. 1.

As mentioned above, the spectral response (zero order transmission) of a single layer is depicted in Fig. 1. The maximum of transmission is located near the cut-off wavelength $\lambda_{c}^{\mathrm{TE}_{11}}=$ $926.5 \mathrm{~nm}$ and reaches $88 \%$. The corresponding peak is relatively wide and shows a small but a non zero transmission for wavelengths greater than the cut-off. This property is of big importance. In fact, this small transmission, which corresponds to a very large reflection, can be increased, as classically, by the use of a FabryPérot (FP) interferometer.

The FP interferometer is designed by cascading two metallic layers as presented in Fig. 2. Fig. 3 shows the transmission spectrum of the double structure where the distance between the two metallic layers is fixed to $d=600 \mathrm{~nm}$. Two peaks, which are

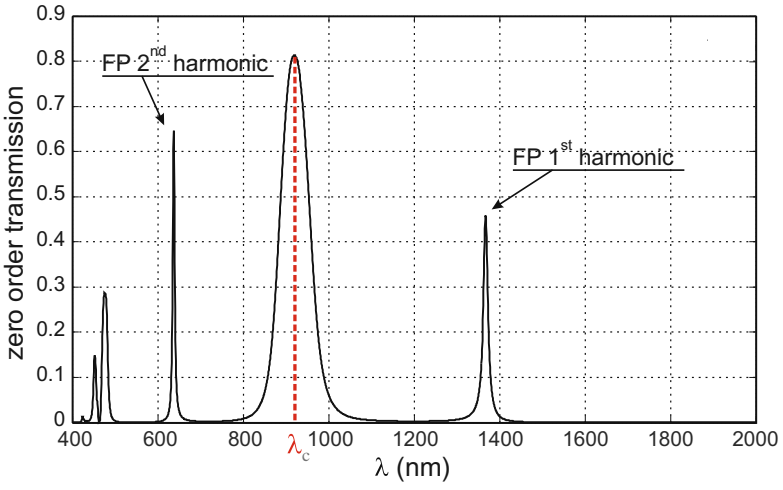

Fig. 3. FDTD computed transmission spectrum of the structure presented on Fig. 2 for $p=350 \mathrm{~nm}, R_{i}=75, R_{o}=100, h=100$ and $d=600 \mathrm{~nm}$.

FP harmonics, appear in the transmission spectrum in addition to the wide one previously observed with the single structure. These peaks do not only appear for wavelengths smaller than the cut-off but also beyond $\lambda_{c}^{\mathrm{TE}_{11}}$.

It is interesting to note that around the cut-off, the transmission is due to the guided mode inside the apertures and should appear whatever the FP thickness is. Therefore, its value is equal to the square of the transmission of a single layer. All these explanations are valid in the case there is no FP harmonic near the cut-off.

To confirm these statements, we computed the transmission spectra for a range of values of $d$ varying from 100 to $600 \mathrm{~nm}$ (see Fig. 4). As described above, the two FP peaks are visible and their positions depend on the FP thickness whereas the wide peak always occurs at the cut-off. When $d$ varies, the positions of the two peaks change according to the law of phase matching $2 \sqrt{\varepsilon} e=m \lambda$ where $e$ is the effective thickness of the FP and $m \in \mathbb{Z}$. As it will be discussed further, the value of $e$ depends both on the nature of the metal and on the geometrical parameters of the apertures.

If FP peak is close from the cut-off, there is a strong coupling between the two phenomena namely the guided mode and the FP resonance as demonstrated in Fig. 4. In Fig. 4, this coupling can be seen for the first FP harmonic: the FP peak crosses the one of the guided mode as the thickness $d$ is increased. This coupling leads to a degeneracy breaking, i.e. the apparition of two supplementary states (two

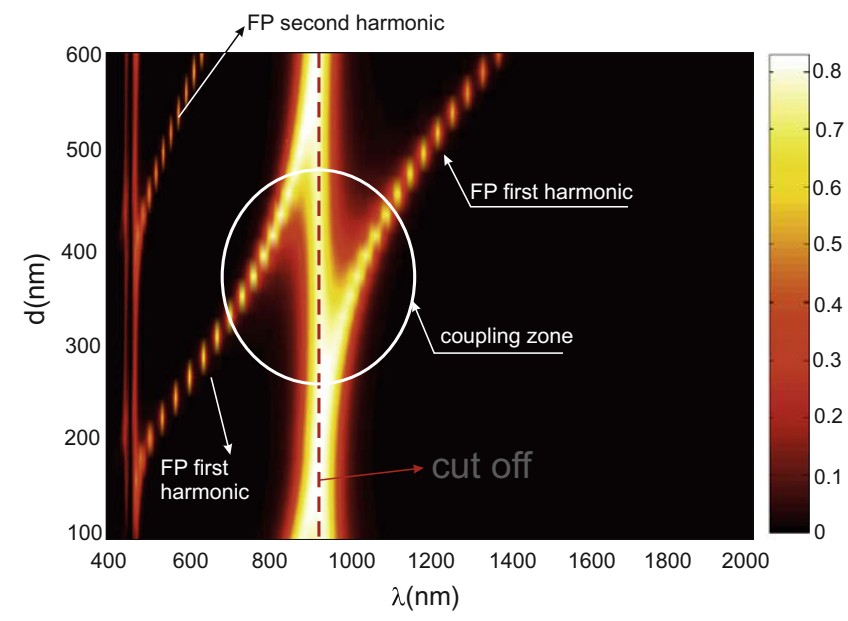

Fig. 4. FDTD computed transmission in color level versus the wavelength and the distance between the two metallic nano-structured layers. The red dashed line corresponds to the cut-off wavelength of the $\mathrm{TE}_{11}$ guided mode. The discontinuities seen on the FP peaks come from the discrete values of $d$ used in the calculations (the numerical step is of $20 \mathrm{~nm}$ ). 
transmission peaks) in addition to the $\mathrm{TE}_{11}$ guided mode that is always present. Far from the guided modes, the FP peak positions recover their usual values governed by the classical phase-matching FP law.

\section{Semi-analytical approach and interpretation}

In order to gain a more physical insight, a semi-analytical approach for the calculation of the transmission through the double structure is given. As for a conventional FP, the normalized transmitted energy can be written as:

$$
T^{\mathrm{FP}}(\lambda)=\frac{T(\lambda)^{2}}{[1-R(\lambda)]^{2}+4 R(\lambda) \sin ^{2}\left(\frac{\phi}{2}\right)},
$$

where $T(\lambda)$ and $R(\lambda)$ are the energy transmission and reflection coefficients of the single metallic nano-structured layer of which the FP is composed. $\phi$ is the phase associated with the distance between the two layers. At normal incidence, its value is given by:

$\phi=\frac{4 \pi \sqrt{\varepsilon} e(\lambda)}{\lambda}$.

As it is well known, a small transmission $T(\lambda)$ of a single layer (i.e. large reflection coefficient $R(\lambda)$ ) leads, in the case of two layers, to a large value of the finesse $\mathscr{F}(\lambda)$ [14]. The variations versus the wavelength of these three quantities are shown in Fig. 5. In addition, the absorption $(A(\lambda)=1-R(\lambda)-T(\lambda))$ is also reported and it is found to be negligible except around the cut-off. Consequently, a FP based on two of such layers will lead to high transmission at its resonances.

Note here that with conventional FP high values of peak transmission and finesse are generally incompatible and a compromise must be found. Thus, one can expect a finesse around 100 but the transmission is then around 0.2 (See Table XV on page 333 of Ref. [15]). However, the proposed double structure permits to reach a high finesse (200) together with large transmission peaks (0.5). This property is directly linked to the reflection and transmission coefficients of the single structured silver film given in Fig. 5. In other words, the annular cavities seem to increase the decay length of the evanescent field that goes through the metallic plate compared to a homogeneous silver film.

From Fig. 5, it is clear that at the cut-off wavelength the finesse has a very small value because no FP resonance occurs; the light directly channels through the two metallic layers as a guided wave inside the annular apertures $(R=0)$.

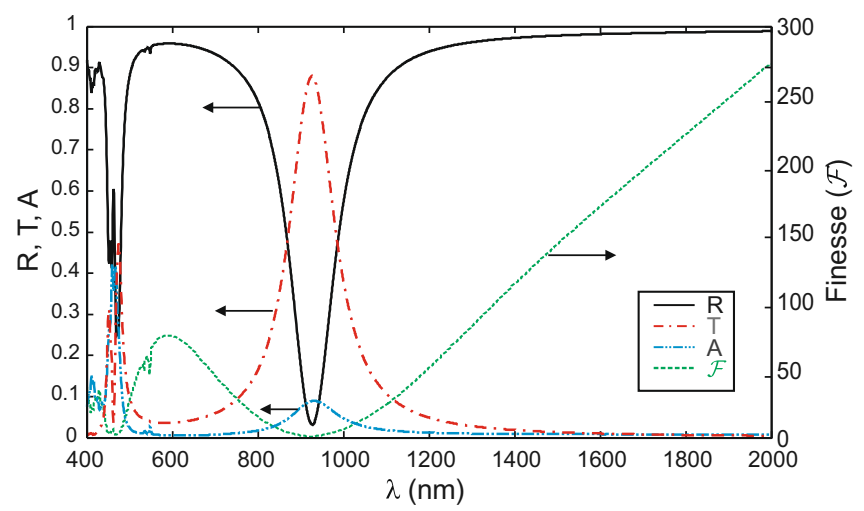

Fig. 5. Transmission (red dotted dashed line), reflection (black solid line) and absorption (blue dotted-dotted dashed line) spectra through a single metallic layer perforated with annular apertures arranged into a square array. The period is $p=350 \mathrm{~nm}$, the inner and the outer radius of the coaxial cavity are $R_{i}=75$ and $R_{0}=100 \mathrm{~nm}$, respectively. The metal thickness is set to $h=100 \mathrm{~nm}$ and the structure is illuminated by an $x$-linearly polarized plane wave at normal incidence. The finesse of the cascaded structure (dotted green line) is deduced from the reflection coefficient.
Usually, the phase $\phi$ is determined from the geometrical distance $d$ between the two metallic layers. This is valid when the penetration depth $e_{m}$ of light in the metal is very small compared to $d$. However, for small values of $d, e_{m}$ must be taken into account. $e_{m}(\lambda)$ can be easily determined by: $e_{m}(\lambda)=\frac{\lambda}{2 \pi n^{\prime \prime}(\lambda)}$ where $n^{\prime \prime}(\lambda)$ is the imaginary part of the index given also by $n^{\prime \prime}=\operatorname{Im}\left(\sqrt{\varepsilon_{m}(\lambda)}\right), \varepsilon_{m}$ being the dielectric permittivity of the metal. Fig. 6 shows that even if the phase in Eq. 1 is corrected by replacing $e$ with $d+2 e_{m}(\lambda)$, the obtained spectra are still slightly different (red shifted) from those in Fig. 4.

Consequently, the effective thickness of the FP can be written as the sum of three terms:

$e(\lambda)=d+2 e_{m}(\lambda)+2 e_{g}(\lambda)$

where $e_{g}(\lambda)$ is the geometrical penetration depth induced by the nano-structuration of the single metallic layer. By combining the results of Figs. 4 and 6 and the value of $e_{m}(\lambda)$ obtained by using the Drude model [1], $e_{g}(\lambda)$ can be deduced as it is shown in Fig. 7 (red circles). The $e_{g}$ values can also be determined by calculating the

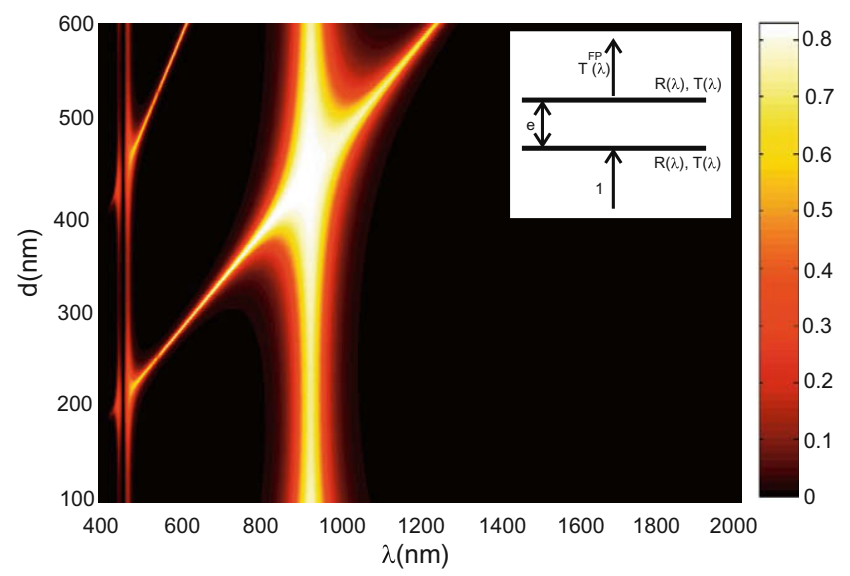

Fig. 6. Calculated transmission from Eq. 1 in color level versus the wavelength and for $d$ varying form 100 to $600 \mathrm{~nm}$. The effective distance $e(\lambda)$ between the two metallic structures is replaced by $e=d+e_{m}(\lambda)$ in Eq. 1 . The inset shows the theoretical schema used to calculate the transmission of the double structure.

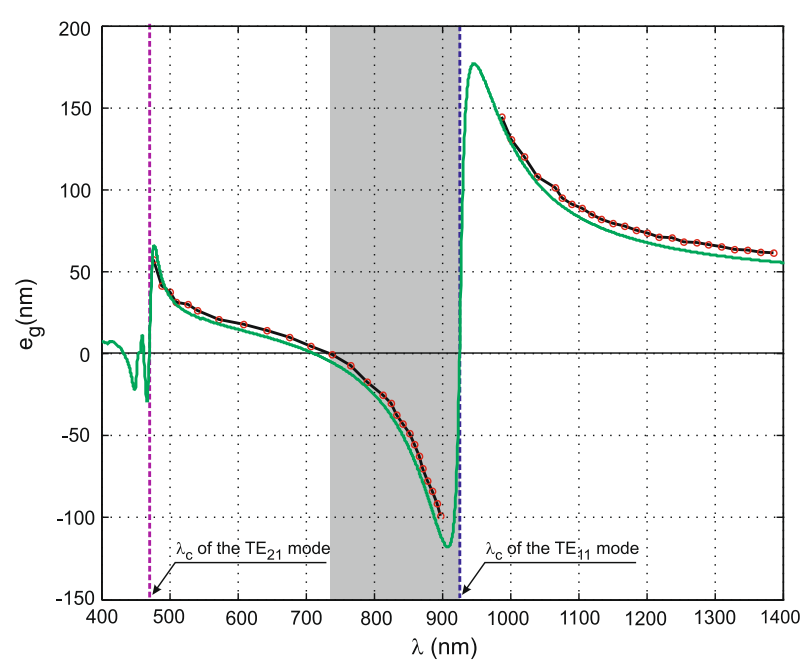

Fig. 7. Geometrical penetration depth of the electromagnetic wave versus the wavelength. The line with red circles is deduced from Eq. (3). The green solid curve is obtained through the FDTD calculations of the reflection phase of a single structured silver film using Eq. 5. The shaded zone corresponds to $e_{g}<0$ which means that the metallic plates induce a negative optical path compared to non structured layers. 


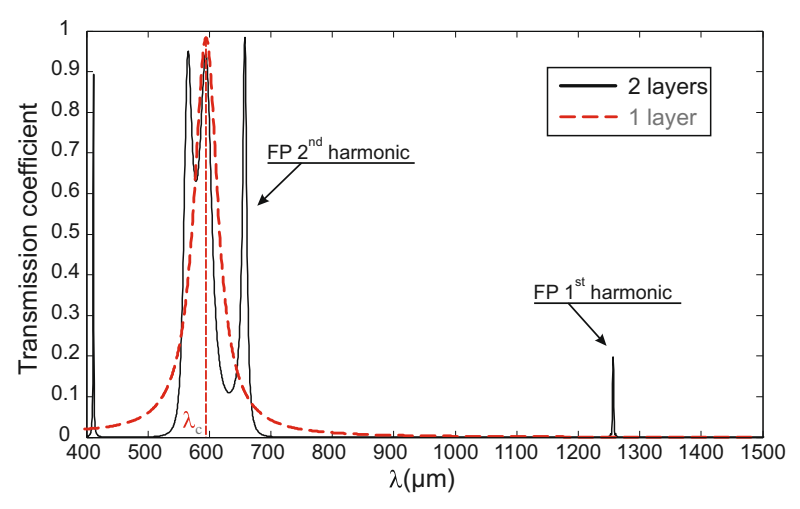

Fig. 8. Transmission spectra of a single structure (dashed red line) and of two cascaded metallic layers (solid black line) in the case of a perfectly conducting metal. For the two calculations, the period is $p=350 \mu \mathrm{m}$, the inner and the outer radius of the coaxial cavity are $R_{i}=75$ and $R_{0}=100 \mu \mathrm{m}$, respectively. The metal thickness is set to $h=100 \mu \mathrm{m}$ and the structure is illuminated by a linearly polarized plane wave at normal incidence. For the cascaded structure, the distance $d$ was fixed to $d=600 \mu \mathrm{m}$. Note that the transmission at the wavelength of the first FP harmonic is enhanced by a factor 150 .

phase $\phi_{R}(\lambda)$ of the reflection coefficient of a single structured silver film. In fact, the phase $\phi$ can be expressed through:

$\phi=\frac{4 \pi d}{\lambda}+2 \phi_{R}(\lambda)$

A specific FDTD simulation is then performed to calculated this phase $\left(\phi_{R}(\lambda)\right)$. Combining Eqs. 3 and 4 one obtain:

$e_{g}(\lambda)=\frac{\lambda \phi_{R}(\lambda)}{2 \pi}-e_{m}(\lambda)$.

The obtained values (green solid curve) are plotted on the same Fig. 7: there is a good agreement with the discrete data obtained from Figs. 4 and 6 . The small discrepancy is due to the spatial mesh used in the FDTD algorithm. Note that $e_{g}$ becomes negative for $\lambda \in\left[733 \mathrm{~nm}, \lambda_{c}^{\mathrm{TE}_{11}}\right]$. In fact the effective index of the guided mode can be smaller than 1 [16] that leads, obviously, to $e_{g}<0$.

For the right part of the curve $\left(\lambda>\lambda_{c}^{\mathrm{TE}_{11}}\right)$, there is no guided mode but the light passes through one metallic layer by tunnel effect and thus, through the whole structure by resonant tunnel effect [17]. In this case, the metallic layer acts as a homogeneous layer with effective permittivity and thickness. Consequently, the penetration depth decays exponentially with the wavelength.

It is of fundamental importance to note that the previous behavior is not sensitive to the lateral shifts $L_{x}$ and $L_{y}$ (see Fig. 2) between the two layers. Computations, not reported here, fortunately show that varying $L_{x}$ and $L_{y}$ from 0 to $p / 2$ does not affect at all the transmission spectrum. Then, the alignment of the AAA layers is not a technological constraint for the fabrication of such a device. This property is only valid if the two layers are far enough so that no near-field coupling occurs.

\section{The perfect conductor case}

To demonstrate the potentiality of this structure, we extend the present study to other electromagnetic domains by considering the case of AAAs made in perfect conductors. This corresponds to metals in the far infrared, terahertz or microwave domains. Fig. 8 shows the transmission spectrum through the same structure when silver is replaced by a perfect conductor and when dimensions are in microns instead of nanometers.

Because the metal used is lossless, the transmission can reach $100 \%$. If we cascade two structures, the transmission behavior shows additional peaks corresponding to the harmonics of the equivalent $\mathrm{FP}$ interferometer.
As in the case of a real metal, peaks can appear far beyond the cut-off wavelength of the guided mode. The position of the wide peak (located at $\lambda_{c}^{\mathrm{TE}_{11}}$ ) is independent of the distance between the two metallic layers whereas the positions of the FP peaks depend on the distance $d$. It is also obvious that for perfect conductor $e_{m}(\lambda)$ is equal to zero. Therefore, the discrepancy between $d$ and $e$, that occurs also in the case of perfect conductor metals, is only due to the additional geometrical penetration depth $e_{g}(\lambda)$ that can be also determined from Eq. (3). For example, Fig. 8 leads to $e_{g}=60 \mu \mathrm{m}$ at $\lambda=1260 \mu \mathrm{m}$.

\section{Conclusion}

In summary, we proposed an original structure based on subwavelength apertures engraved into optically thick metallic films that allows simultaneously high and sharp transmission peaks. A complete study of the transmission through these cascaded AAAs has been presented and the mechanisms involved in the electromagnetic interactions have been elucidated. The results based on a numerical method (FDTD method) show a good agreement with a semi-analytical model. Many applications can be founded upon this promising structure both in the optical and $\mathrm{THz}$ domains. For the visible range, this cascaded AAA structure can be used to design submicrometric interferometers with very high $Q$ factor or to build nanometric-sized modulators by replacing the vacuum between the two metallic layers by an electro-optical material such as lithium niobate. In the $\mathrm{THz}$ range, this device can also be used to enhance the emission of quantum-cascade lasers $[18,19]$. Other applications for Radome-Radar, spectroscopic detection or flat screens can also be designed from this interesting structure.

\section{Acknowledgement}

This work has been partially supported by C'Nano GE, the Pôle de Compétences en Nanosciences Grand Est.

\section{References}

[1] F.I. Baida, D. Van Labeke, Opt. Commun. 209 (2002) 17.

[2] F.I. Baida, D. Van Labeke, Phys. Rev. B 67 (2003) 155314.

[3] F.I. Baida, D. Van Labeke, G. Granet, A. Moreau, A. Belkhir, Appl. Phys. B 79 (2004) 1.

[4] W. Fan, S. Zhang, B.0. Minhas, K.-J. Malloy, S.R.J. Brueck, Phys. Rev. Lett. 94 (2005) 033902.

[5] J. Salvi, M. Roussey, F.I. Baida, M.-P. Bernal, A. Mussot, T. Sylvestre, H. Maillotte, D. Van Labeke, A. Perentes, I. Utke, C. Sandu, P. Hoffmann, B. Dwir, Opt. Lett. 30 (2005) 1611 .

[6] A. Perentes, I. Utke, B. Dwir, M. Leutenegger, T. Lasser, P. Hoffmann, F. Baida, M.-P. Bernal, M. Roussey, J. Salvi, D. Van Labeke, Nanotechnology 16 (2005) S273.

[7] Y. Poujet, M. Roussey, J. Salvi, F.I. Baida, D. Van Labeke, A. Perentes, C. Santschi, P. Hoffmann, Photon. Nanostruct. 4 (2006) 47.

[8] J. Rybczynski, K. Kempa, A. Herczynski, Y. Wang, M.J. Naughton, Z.F. Ren, Z.P. Huang, D. Cai, M. Giersig, Appl. Phys. Lett. 90 (2007) 021104.

[9] D. Van Labeke, D. Gérard, B. Guizal, F.I. Baida, L. Li, An angle-independent frequency selective surface in the optical range, Opt. Express 14 (2006) 11945

[10] Q. Cao, P. Lalanne, Phys. Rev. Lett. 88 (2002) 057403.

[11] G.D. Holah, S.D. Smith, J. Phys. E 10 (1977) 101.

[12] Y.-H. Ye, J.-Y. Zhang, Opt. Lett. 30 (2005) 1521.

[13] H.B. Chan, Z. Marcet, K. Woo, D.B. Tanner, D.W. Carr, J.E. Bower, R.A. Cirelli, E. Ferry, F. Klemens, J. Miner, C.S. Pai, J.A. Taylor, Opt. Lett. 31 (2006) 516.

[14] The finesse $\mathscr{F}(\lambda)$ is calculated, as for a conventional Fabry-Pérot, by its rigorous expression: $\mathscr{F}^{-1}(\lambda)=\frac{2}{\pi} \arcsin \frac{[1-R(\lambda)]}{2 \sqrt{R(\lambda)}}$ which leads for large values of $R(\lambda)$ to $\mathscr{F}(\lambda)=\frac{\pi \sqrt{R(\lambda)}}{1-R(\lambda)}$.

[15] M. Born, E. Wolf, Interference and Diffraction of Light, Cambridge University Press, 1997.

[16] F.I. Baida, A. Belkhir, D. Van Labeke, O. Lamrous, Phys. Rev. B 74 (2006) 205419

[17] J.M. Vigoureux, F. Baida, Opt. Commun. 101 (1993) 297.

[18] B. Williams, S. Kumar, H. Callebaut, Q. Hu, J. Reno, Appl. Phys. Lett. 83 (2003) 2124.

[19] G. Fasching, A. Benz, K. Unterrainer, R. Zobl, A.M. Andrews, T. Roch, W. Schrenk, G. Strasser, Appl. Phys. Lett. 87 (2005) 211112. 